\title{
An Order Four Continuous Numerical Method for Solving General Second Order Ordinary Differential Equations
}

\author{
F. O. Obarhua*, O. J. Adegboro \\ Department of Mathematical Sciences, The Federal University of Technology, Akure, Nigeria
}

\begin{abstract}
Continuous hybrid methods are now recognized as efficient numerical methods for problems whose solutions have finite domains or cannot be solved analytically. In this work, the continuous hybrid numerical method for the solution of general second order initial value problems of ordinary differential equations is considered. The method of collocation of the differential system arising from the approximate solution to the problem is adopted using the power series as a basis function. The method is zero stable, consistent, convergent. It is suitable for both non-stiff and mildly-stiff problems and results were found to compete favorably with the existing methods in terms of accuracy.
\end{abstract}

DOI:10.46481/jnsps.2021.150

Keywords: Numerical Scheme, Continuous hybrid method, Zero stability, Linear and nonlinear

Article History :

Received: 05 January 2021

Received in revised form: 31 January 2020

Accepted for publication: 04 February 2021

Published: 27 February 2021

(C)2021 Journal of the Nigerian Society of Physical Sciences. All rights reserved. Communicated by: B. J. Falaye

\section{Introduction}

We consider the second order Ordinary Differential Equation (ODEs)

$$
y^{\prime \prime}=f\left(x, y, y^{\prime}\right), y(\mu)=\omega_{0}, y^{\prime}(\mu)=\omega_{1}
$$

Equation (1) occur virtually every areas of physical or biological process in connection with numerous problems that are encountered in various aspects of everyday life. It is well conceived that this type of equation can either be solved directly or solved by reducing to system of first order differential equations before applying different methods available to solve the resulting system of first order ODEs Chan et al. [1], Gholamtabar and Parandin [2].

${ }^{*}$ Corresponding author tel. no: +2348062488066

Email address: obarhuaf o@futa.edu.ng (F. O. Obarhua )
Various linear multistep methods with different order of accuracy have been developed for the solution of 1 which varies from discrete linear multistep methods to continuous linear multistep methods. Lambert and Watsan, [3] reported that linear multistep methods generally are more efficient in terms of accuracy with weak stability properties for a given number of evaluation per step and suffered the disadvantage of requiring additional starting values and special procedures for changing step length $h$. It is also good to note that continuous linear multistep methods have advantages over the discrete methods in such a way that they give better error estimation, they provide a simplified form of coefficients for further evaluation at different points, and they provide solutions at all interior points within the interval of integration Saravi and Mirrajei, [4], Kayode and Awoyemi, [5], Golbabai and Arabshahi [6]. Among the first methods developed are first derivative methods that are implemented in predictor-corrector mode, and Taylor series expan- 
sion are adopted to provide the starting values. The identified setbacks of the predictor-corrector methods are; they are very costly to implement and reduced order of accuracy of the predictors. Recently, authors have proposed different methods of higher order differential equations to improve on the existing setbacks. Such improved methods are Kayode and Adeyeye, $[7,8]$ and Kayode and Obarhua, $[9,10]$. They independently proposed linear multistep methods of higher order of accuracies and same order of main predictors and the correctors and hence improved significantly the accuracies of the methods.

This work proposed an accurate continuous numerical hybrid method for direct solution of initial value problems of ODEs. The derived method is capable to handle stiff, mildly stiff, nonlinear and engineering problems modeled as a second order initial value problem of ODEs.

\section{Derivation of the Method}

We define the general power series approximation solution in the form

$$
\begin{aligned}
& y(x)=\sum_{j=0}^{(c+i)-1} a_{j} x^{j} \\
& y^{\prime}(x)=\sum_{j=1}^{(c+i)-1} j a_{j} x^{j-1} \\
& y^{\prime \prime}(x)=\sum_{j=2}^{(c+i)-1} j(j-1) a_{j} x^{j-2}
\end{aligned}
$$

Equating (4) with (1) gives

$$
f\left(x, y, y^{\prime}\right)=\sum_{j=2}^{(c+i)-1} j(j-1) a_{j} x^{j-2}
$$

Equation (2) is interpolated at $x_{n+i}, i=2, \frac{5}{2}$ and (5) is collocated at $x_{n+c}, c=0(1) 3$.

Therefore, interpolation and collocation equation at the selected grid and offstep points give rise to system of equations which can be express in matrix form

$$
\left(\begin{array}{cccccc}
1 & x_{n+2 h} & x_{n+2 h}^{2} & x_{n+2 h}^{3} & x_{n+2 h}^{4} & x_{n+2 h}^{5} \\
1 & x_{n+r h} & x_{n+r h}^{2} & x_{n+r h}^{3} & x_{n+r h}^{4} & x_{n+r h}^{5} \\
0 & 0 & 2 & 6 x_{n} & 12 x_{n}^{2} & 20 x_{n}^{3} \\
0 & 0 & 2 & 6 x_{n+h} & 12 x_{n+h}^{2} & 20 x_{n+h}^{3} \\
0 & 0 & 2 & 6 x_{n+2 h} & 12 x_{n+2 h}^{2} & 20 x_{n+2 h}^{3} \\
0 & 0 & 2 & 6 x_{n+3 h} & 12 x_{n+3 h}^{2} & 20 x_{n+3 h}^{3}
\end{array}\right)\left(\begin{array}{l}
a_{0} \\
a_{1} \\
a_{2} \\
a_{3} \\
a_{4} \\
a_{5}
\end{array}\right)=\left(\begin{array}{l}
y_{n+2} \\
y_{n+r} \\
f_{n} \\
f_{n+1} \\
f_{n+2} \\
f_{n+3}
\end{array}\right)
$$

Applying transformation $t=\frac{1}{h}\left(x-x_{n+k-1}\right), \quad k=3, t=(0,1]$
in Obarhua [11], the continuous coefficients are obtained as follows

$$
\begin{aligned}
\alpha_{2} & =-\left(\frac{-r h+t h+2 h}{h(r-2)}\right) \\
\alpha_{r} & =\frac{t h}{h(r-2)} \\
\beta_{0} & =\frac{h^{5}}{360}\left(\frac{-3 t^{5}+10 t^{3}+8 t+3 t r^{4}-24 t r^{3}+62 t r^{2}-56 t r}{h^{3}}\right) \\
\beta_{1} & =-\frac{h^{5}}{120}\left(\frac{-3 t^{5}-5 t^{4}+20 t^{3}-72 t+3 t r^{4}-19 t r^{3}+22 t r^{2}+44 t r}{h^{3}}\right) \\
\beta_{2} & =\frac{h^{5}}{120}\left(\frac{-3 t^{5}-10 t^{4}+10 t^{3}+60 t^{2}+48 t+3 t^{4} r^{4}}{h^{3}}\right. \\
& \left.\frac{-14 t^{4} r^{3}+2 t^{4} r^{2}+4 t r}{h^{3}}\right)
\end{aligned}
$$

The first derivatives of equation (7) are

$$
\begin{aligned}
\alpha_{2}^{\prime} & =-\frac{1}{(r-2)} \\
\alpha_{r}^{\prime} & =\frac{1}{(r-2)} \\
\beta_{0}^{\prime} & =\frac{h^{5}}{360}\left(\frac{-15 t^{4}+30 t^{2}+3 r^{4}-24 r^{3}+62 r^{3}-56 r+8}{h^{3}}\right) \\
\beta_{1}^{\prime} & =-\frac{h^{5}}{120}\left(\frac{-15 t^{4}-20 t^{3}+60 t^{2}+3 r^{4}-19 r^{3}+22 r^{2}+44 r-72}{h^{3}}\right) \\
\beta_{2}^{\prime} & =\frac{h^{5}}{120}\left(\frac{-15 t^{4}-40 t^{3}+30 t^{2}+120 t+12 t^{3} r^{4}-56 t^{2} r^{3}+8 t^{3} r^{2}}{h^{3}}\right. \\
& \left.+\frac{4 r+48}{h^{3}}\right) \\
\beta_{3}^{\prime} & =-\frac{h^{5}}{360}\left(\frac{-15 t^{4}-60 t^{3}-60 t^{2}+3 r^{4}-9 r^{3}+8 t^{3} r^{2}+16 t^{3} r+8}{h^{3}}\right)
\end{aligned}
$$

Evaluating equation (7) and (8) at $t=1$ yield the discrete order continuous numerical scheme

$$
\begin{aligned}
y_{n+3} & =-\frac{1}{360(r-2)}\left(-60 h^{2} r^{5} f_{n+2}-360 y_{n+2}-630 h^{2} f_{n+2}\right. \\
& -30 h^{2} f_{n}-3 h^{2} r^{5} f_{n+3}+9 h^{2} r^{5} f_{n+2}-9 h^{2} r^{5} f_{n+1}+3 h^{2} r^{5} f_{n} \\
& +15 h^{2} r^{4} f_{n+3}-20 h^{2} r^{3} f_{n+3}-180 h^{2} r^{3} f_{n+1}+90 h^{2} r^{3} f_{n+2} \\
(6) & -180 h^{2} r^{2} f_{n}+110 h^{2} r^{3} f_{n}-30 h^{2} r^{4} f_{n}+75 h^{2} r^{4} f_{n+1} \\
& -60 h^{2} r^{4} f_{n+2}+360 r y_{n+2}+291 h^{2} r f_{n+2}+360 y_{n+r}-360 h^{2} f_{n+1} \\
& \left.+38 h^{2} r f_{n+3}+444 h^{2} r f_{n+1}+127 h^{2} r f_{n}\right)
\end{aligned}
$$

Gaussian elimination method is then applied to solve equation (6) to obtain the unknown coefficients $a_{j}^{\prime} s$ which is then substituted into (2). Continuous system is obtained after some algebraic simplifications. 
its first derivative is given as

$$
\begin{aligned}
y_{n+3}^{\prime} & =-\frac{1}{360 h(r-2)}\left(9 h^{2} r^{5} f_{n+2}+9 h^{2} r^{5} f_{n+1}+3 h^{2} r^{5} f_{n}+90 h^{2} r^{3} f_{n+2}\right. \\
& -60 h^{2} r^{4} f_{n+2}+110 h^{2} r^{3} f_{n}-180 h^{2} r^{3} f_{n+1}-180 h^{2} r^{2} f_{n} \\
& -30 h^{2} r^{4} f_{n}+75 h^{2} r^{4} f_{n+1}-3 h^{2} r^{5} f_{n+3}-20 h^{2} r^{3} f_{n+3} \\
& +15 h^{2} r^{4} f_{n+3}-254 h^{2} f_{n+3}-858 h^{2} f_{n+2}-282 h^{2} f_{n+1} \\
& -46 h^{2} f_{n}+360 y_{n+r}+405 h^{2} r f_{n+2}+405 h^{2} r f_{n+1}+135 h^{2} r f_{n+3} \\
& \left.+135 h^{2} r f_{n}-360 y_{n+2}\right)
\end{aligned}
$$

The values of $r$ is taken in the interval $r \in(2,3)$ to obtain a particular discrete hybrid method. For the purpose of testing the properties of equation (9), the value of $r$ is taken to $\frac{5}{2}$ to have

$$
y_{n+3}=2 y_{n+\frac{5}{2}}-y_{n+2}+\frac{h^{2}}{384}\left(33 f_{n+3}+83 f_{n+2}-25 f_{n+1}+5 f_{n}\right)(1
$$

with its first derivative given by

$$
\begin{aligned}
h y_{n+3}^{\prime}= & 2 y_{n+\frac{5}{2}}-2 y_{n+2}+\frac{h}{5760}\left(2047 f_{n+3}\right. \\
& \left.+3069 f_{n+2}-999 f_{n+1}+203 f_{n}\right)
\end{aligned}
$$

\section{Implementation of the Method (11)}

In order to implement the implicit linear one-point discrete scheme (11) and its derivative (12), the symmetric explicit schemes and their derivatives are also developed by the same procedure for the evaluation of $y_{n+3}$ and $y_{n+3}^{\prime}$ contained in $f_{n+3}$ in the main scheme (11) and its derivative (12).

$$
y_{n+3}=2 y_{n+\frac{5}{2}}-y_{n+1}+\frac{h^{2}}{240}\left(66 f_{n+\frac{5}{2}}-10 f_{n+2}+5 f_{n+1}-f_{n}\right)(
$$

and its first derivative as

$$
\begin{aligned}
h y_{n+3}^{\prime} & =-2 h y_{n+\frac{5}{2}}+2 h y_{n+1} \\
& +\frac{h^{2}}{3600}\left(-4094 f_{n+\frac{5}{2}}+1920 f_{n+2}-655 f_{n+1}+129 f_{n}\right)
\end{aligned}
$$

Other explicit schemes were also generated to evaluate other starting values using Taylor series expansions to evaluate the values for $y_{n+i}, y_{n+i}^{\prime}$ as

$$
y_{n+i}=y_{n}+(j h) y_{n}^{\prime}+\frac{(j h)^{2}}{2 !} f_{n}+\frac{(j h)^{3}}{3 !}\left(\frac{\partial f_{n}}{\partial x_{n}}+y_{n}^{\prime} \frac{\partial f_{n}}{\partial y_{n}}+f_{n} \frac{\partial f_{n}}{\partial y_{n}^{\prime}}\right)+o\left(h^{4}\right)(
$$

tion 1 and the interval of periodicity, Lambert and Watson [3] introduced the following scalar test problem as

$$
y^{\prime \prime}=-\lambda^{2} y
$$

Based on the theory developed in Lambert and Watson [3], when multistep method

$$
\sum_{j=0}^{l} \alpha_{j} y_{n+j}=h^{2} \sum_{j=0}^{l} \theta_{j} f_{n+j},
$$

is applied to the scalar test equation (17), a difference equation of the form

$$
\sum_{i=0}^{l}\left(\alpha_{i}+H^{2} \theta_{i}\right) y_{n+i}=0
$$

is obtained, where $H=p h, \quad h$ is the step length and $y_{n}$ is the computed approximation to $y\left(x_{0}+n h\right), \quad n=0,1,2, \ldots$. Then, we have following definitions.

Definition. (See Konguetsof and Simos, [12]) Numerical method (19) has an interval of periodicity $\left(0, H_{0}^{2}\right)$, if $\forall H^{2} \in$ $\left(0, H_{0}^{2}\right), Q_{i}, \quad i=1(1) l$ satisfy

$$
\left|Q_{1}\right|=\left|Q_{2}\right|=1, \quad\left|Q_{j}\right| \leq 1, \quad j=3(1) l .
$$

Definition. Following [3], a numerical method is P-stable if its interval of periodicity is $(0, \infty)$. Therefore, we obtain the interval of periodicity of the new method, which is Equal to $(0,-2.4)$ and the stability domain of the method is as shown in Figure 1.

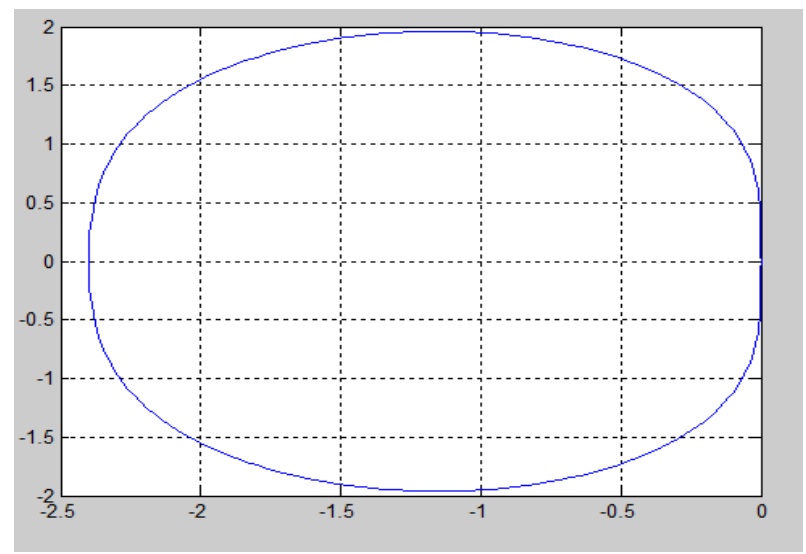

Figure 1: The stability domain of the new method

\subsection{Order and Error Constant of the Method}

$$
y_{n+i}^{\prime}=y_{n}^{\prime}+(j h) f_{n}+\frac{(j h)^{2}}{2 !}\left(\frac{\partial f_{n}}{\partial x_{n}}+y_{n}^{\prime} \frac{\partial f_{n}}{\partial y_{n}}+f_{n} \frac{\partial f_{n}}{\partial y_{n}^{\prime}}\right)+o\left(h^{4}\right)(16)
$$

The method proposed by Lambert (1973) in Olanegan et al. [13] is adopted in this paper, with linear operator:

$$
\sum_{j=0}^{k} \alpha_{j} y_{n+j}=h^{2} \sum_{j=0}^{k} \beta_{j} f_{n+j}
$$

\subsection{Region of Absolute Stability}

In other to investigate the periodic stability properties of the numerical methods for solving the initial-value problem equa- 
We associate the linear operator $L$ with the scheme and defined as

$$
L\{y(x), h\}=\sum_{j=0}^{k}\left[\alpha_{j} y(x+j h)-h^{2} \beta_{j} y^{\prime \prime}(x+j h)\right]
$$

Where $\alpha_{0}$ and $\beta_{0}$ are both non-zero and $y(x)$ is an arbitrary function which is continuous and differentiable on the interval $[a, b]$. Expanding the form $y(x)$ and $y^{\prime \prime}(x)$ in Taylor series and comparing coefficients of $h$, we obtained

$$
\begin{aligned}
\Delta[y(x) ; h] & =C_{0} y(x)+C_{1} h y^{\prime}(x)+\cdots+C_{p} h^{p} y^{p}(x) \\
& +C_{p+1} h^{p+1} y^{p+1}(x)+C_{p+2} h^{p+2} y^{p+2}(x)+\cdots
\end{aligned}
$$

The method (11) and its associate linear difference operator (13) are said to have order $p$ if $c_{0}=c_{1}=\cdots=c_{p+1}=0$ and $c_{p+2} \neq 0$. The value $c_{p+2}$ is called error constant. Therefore, in this paper, the method (11) is of order 4 and the error constant $c_{p+2}=-\frac{21}{2560}$ or $-8.2031 \times 10^{-3}$ and its derivative (12) is of order 4 and error constant $c_{p+2}=-\frac{35}{1536}$ or $-2.2786 \times 10^{-2}$.

\subsection{Consistency of the Method}

A numerical method is said to be consistent if the following conditions are satisfied

1. The order of the method must be greater or equal to 1 , $p \geq 1$.

2. $\sum_{j=0}^{k} \alpha_{j}=0$

3. $\rho(r)=\rho^{\prime}(r)=0$

4. $\rho^{\prime \prime}(r)=2 ! \sigma(r)$

Where $\rho(r)$ and $\sigma(r)$ are the first and second characteristics polynomial of our method. according to Adesanya et al. [14], the first condition is a sufficient condition for a method to be consistent. Since our method is of order 4 then it is consistent.

\subsection{Zero Stability}

Since $\left|z_{i}\right|=|0,0,1| \leq 1$ the method is zero stable.

\subsection{Convergence}

A method is said to be convergent if and only if it is consistent and zero stable, hence our method is convergent.

\section{Numerical Examples}

The method is applied to solve the following linear and nonlinear second order initial value problems of ordinary differential equations directly without reduction to system of first order equations.

Problem 1: $y^{\prime \prime}=y^{\prime}, \quad y(0)=0 ; \quad y^{\prime}(0)=-1 ; \quad h=0.1$

Theoretical solution: $y(x)=1-e^{x}$

This problem has been used in Kayode and Adeyeye [8] of order six to check the behavior of the methods. Table 1 shows the absolute errors of the methods for the purpose of comparison.
The obtained results in the Table give the good performance of the proposed method.

Problem 2: $y^{\prime \prime}+1001 y^{\prime}+1000 y=0, \quad y(0)=1 ; \quad y^{\prime}(0)=$ $-1 ; h=0.05$

Theoretical solution: $y(x)=e^{-x}$

The Problem 2 was solved by Anake [15] of order 4. The new method was applied to solve it for the purpose of comparison. The results are shown in Table 2.

Problem 3: $y^{\prime \prime}=100 y^{\prime}, \quad y(0)=1 ; \quad y^{\prime}(0)=-10 ; \quad h=0.01$

Theoretical solution: $y(x)=e^{-10 x}$

Table shows the absolute errors at different points of the integration interval when $h=0.01$ was solved by Awari [16] of order five. The new method was applied to solve it for the purpose of comparison. The results show that the proposed method performed better than Awari [16].

Problem 4: $\quad y^{\prime \prime}-x\left(y^{\prime}\right)^{2}=0, \quad y(0)=1 ; \quad y^{\prime}(0)=\frac{1}{2} ; \quad h=$ 0.003125

Theoretical solution: $y(x)=1+\frac{1}{2} \ln \left[\frac{2+x}{2-x}\right]$

We have solved this problem with the new method and the results have been compared with Kayode and Adeyeye [7] of order six shown in Table 5.

\section{Problem 5:}

\section{Resonance Vibration of a Machine}

A stamping machine applies hammering forces on metal sheets by a die attached to the plunger moves vertically up and down by a fly wheel spinning at constant set speed. The constant rotational speed of the fly wheel makes the impact force on the sheet metal, and therefore the supporting base, intermittent and cyclic. The bearing base on which the metal sheet is situated has a mass, $M=2000 \mathrm{~kg}$. The force acting on the base follows a function: $F(t)=2000 \sin (10 t)$, in which $t$-time in seconds. The base is supported by an elastic pad with an equivalent spring constant $k=2 \times 10^{5} \mathrm{~N} / \mathrm{m}$. Determine the differential equation for the instantaneous position of the base $y(t)$ if the base is initially depressed down by an amount $0.1 \mathrm{~m}$.

Solution: The mass-spring system above is modeled as differential equation as:

The Bearing base mass $=2000 \mathrm{~kg}$

Spring constant $k=2 \times 10^{5} \mathrm{~N} / \mathrm{m}$.

Force $(m a)$ on the metal sheet $=m \frac{d^{2} y}{d t^{2}}=m y^{\prime \prime}$ i.e. $m a=m y^{\prime \prime}=2000 \sin (10 t)$; where $a=y^{\prime \prime}$ Initial conditions on the system are $y\left(t_{0}\right)=y_{0} ;\left.\frac{d y}{d t}\right|_{t=o}=y^{\prime}\left(t_{0}\right)=y_{0}^{\prime} ; t_{0}=$ $0, y_{0}^{\prime}=0.1$

Therefore, the governing equation for the instantaneous position of the base $y(t)$ is given by

$$
\begin{aligned}
& M y^{\prime \prime}+k y=F(t) ; \quad y\left(t_{0}\right)=y_{0}, \quad y^{\prime}\left(t_{0}\right)=y_{0}^{\prime} \\
& 2000 y^{\prime \prime}+2 \times 10^{5} y=2000 \sin 10 t, \quad y^{\prime}(0)=0, y(0)=0.1
\end{aligned}
$$

Theoretical solution: $y(t)=\frac{1}{10} \cos 10 t+\frac{1}{200} \sin 10 t-\frac{t}{20} \cos 10 t$

\section{Conclusion}

An order four continuous numerical method for solving general second order ordinary differential equations is proposed and applied to solve directly without reducing to system of first 
Table 1

\begin{tabular}{cccccccccc}
\hline \hline$x$ & 0.2 & 0.3 & 0.4 & 0.5 & 0.6 & 0.7 & 0.8 & 0.9 & 1.0 \\
\hline Error in [8] & $8.17(-7)$ & $3.10(-6)$ & $6.57(-6)$ & $1.14(-5)$ & $1.79(-5)$ & $2.64(-5)$ & $3.72(-5)$ & $5.06(-5)$ & $6.72(-5)$ \\
Error in (11) & $4.25(-8)$ & $7.47(-8)$ & $1.52(-7)$ & $2.45(-7)$ & $3.54(-7)$ & $5.31(-7)$ & $7.37(-7)$ & $9.73(-7)$ & $1.31(-7)$ \\
\hline
\end{tabular}

Table 2

\begin{tabular}{|c|c|c|c|c|c|c|c|c|}
\hline$x$ & 0.1 & 0.2 & 0.3 & 0.4 & 0.5 & 0.6 & 0.7 & 0.8 \\
\hline Error in [15] & $0.10(-9)$ & $0.20(-9)$ & $0.28(-9)$ & $0.34(-9)$ & $0.39(-9)$ & $0.43(-9)$ & $0.45(-9)$ & $0.4(-9)$ \\
\hline Error in (11) & $2.00(-10)$ & $3.15(-10)$ & $2.74(-10)$ & $5.44(-10)$ & $7.53(-10)$ & $2.76(-10)$ & $1.18(-10)$ & $1.76(-10)$ \\
\hline
\end{tabular}

Table 3: Absolute errors at different points of the integration

\begin{tabular}{ccccccccc}
\hline \hline$x$ & 0.01 & 0.02 & 0.03 & 0.04 & 0.05 & 0.06 & 0.07 & 0.08 \\
\hline Error in [16] & $1.15(-7)$ & $3.65(-7)$ & $6.05(-7)$ & $8.50(-7)$ & $1.10(-6)$ & $1.36(-6)$ & $1.45(-6)$ & $1.59(-6)$ \\
Error in (11) & $1.29(-8)$ & $3.01(-8)$ & $5.04(-8)$ & $9.32(-10)$ & $1.40(-7)$ & $1.90(-7)$ & $2.58(-7)$ & $3.32(-7)$ \\
\hline
\end{tabular}

Table 4: Absolute errors for Numerical example 4

\begin{tabular}{cccccccccc}
\hline \hline$x$ & 0.0063 & 0.0094 & 0.0125 & 0.0188 & 0.0250 & 0.0313 & 0.0375 & 0.0437 & 0.0500 \\
\hline Error in (11) & $0.00(0)$ & $0.00(0)$ & $2.81(-14)$ & $2.36(-13)$ & $8.73(-13)$ & $1.91(-12)$ & $2.97(-12)$ & $5.21(-12)$ & $7.55(-12)$ \\
\hline
\end{tabular}

Table 5: Comparison of errors with [7]

\begin{tabular}{ccc}
\hline \hline$x$ & Error in Kayode and Adeyeye [7] & Error in the new Method (11) \\
\hline 0.0063 & $4.831(-11)$ & $0.0000(00)$ \\
0.0094 & $3.382(-9)$ & $0.0000(00)$ \\
0.0125 & $1.580(-8)$ & $2.819(-14)$ \\
0.0156 & $4.333(-8)$ & $1.709(-13)$ \\
0.0188 & $9.391(-8)$ & $2.362(-13)$ \\
\hline
\end{tabular}

Table 6: The new derived method was applied to solve this problem modeled as a second order (IVPs) and it was seen from the results in the Table that the method are useable in Engineering field.

\begin{tabular}{cccc}
\hline \hline & & & \\
$\mathrm{x}$ & Exact solution & Computed solution & Error \\
\hline & & & \\
0.01 & 0.099404629653415691 & 0.099404630038381694 & $3.849660(-10)$ \\
0.02 & 0.097958005773976925 & 0.097958006644224049 & $8.702471(-10)$ \\
0.03 & 0.095207162458893865 & 0.095207165387981033 & $2.929087(-9)$ \\
0.04 & 0.091970827382988077 & 0.091970831862903016 & $4.479915(-9)$ \\
0.05 & 0.087961427477332363 & 0.087961431552930208 & $4.075598(-9)$ \\
0.06 & 0.082363909854646533 & 0.082363917430066838 & $7.575420(-9)$ \\
0.07 & 0.076833743309093400 & 0.076833753917924741 & $1.060883(-9)$ \\
0.08 & 0.069604876901833215 & 0.069604894375183718 & $1.747335(-9)$ \\
0.09 & 0.062811758617177721 & 0.062811776980930309 & $1.836375(-9)$ \\
0.10 & 0.055536073981512724 & 0.055536101603349465 & $2.762184(-8)$ \\
\hline \hline
\end{tabular}

order ordinary differential equations. The method is very flexible and easy to develop and may be applied to solve kinds of second order initial value problems as can be seen in the numerical examples. The method gives a high accuracy when compared the numerical results to the exact solution and a very good performance compared with existing methods in the literature.

\section{References}

[1] R. P. K. Chan, P. Leone, \& A. Tsai, "Order Conditions and Symmetry for Two-Step Hybrid Methods", Int. J. Comp. Math. 81 (2004) 1519.

[2] S. Gholamtabar \& N. Parandin, "Numerical Solutions of Second-Order Differential Equations by Adam Bashforth Method", American J. of Engi. Research 3 (2014) 318. 
[3] J. D. Lambert \& A. Watsan, "A Symmetric Multistep Method for Periodic Initial Value Problem", Journal of the Institute of Mathematics and its Applications 18 (1976) 189.

[4] M. F. Saravi \& S.R. Mirrajei, "Numerical Solution of Linear Ordinary Differential Equations in Quantum Chemistry by Clenshaw Method", World Acad. Sci. Eng. Technol. 49 (2009) 1051.

[5] S. J. Kayode and D.O. Awoyemi, "A Multiple Derivative Collocation Method for Fifth Order Ordinary Differential Equations", J. Math. stat. 6 (2010) 60.

[6] A. Golbabai \& M. M. Arabshahi, "Smart Numerov Generalized Alternating Group Explicit (SNAGE-PR (2)) Scheme for 2-Point P.V. Problem", World Appl. Sci. J. 8 (2010) 267.

[7] S. J. Kayode \& A. Adeyeye, " A 3-Step Hybrid Method for Direct Solution of Second Order Initial Value Problems", Aust. J. of Basic and Applied Sciences 5 (2011) 2121

[8] S. J. Kayode \& A. Adeyeye, "Two-Step Two-Point Hybrid Methods for General Second Order Differential Equations" Afri. J. of Math. \& Compt. Sci. Res. 6 (2013) 191

[9] S. J. Kayode \& F. O. Obarhua, "Continuous $y$ - function Hybrid Methods for Direct Solution of Differential Equations", Intern. J. of Diff. Eq. 12 (2013) 37.

[10] S. J. Kayode \& F. O. Obarhua, "3-Step y-function Hybrid Methods for Di- rect Numerical Integration of Second Order IVPs in ODEs", Theo. Math. \& Appl. 5 (2015) 39.

[11] F. O. Obarhua, "An Efficient Two-Step Symmetric Hybrid Block Method for Solving Second Order Initial Value Problems of Ordinary Differential Equations", Int. J. of Res. and Sci. Innovation 6 (2019) 200.

[12] A. Konguetsof \& T. E. Simos, "An Exponentially Fitted and Trigonometrically Fitted Method for the Numerical Solution of Periodic Initial-Value Problems", Computers and Mathematics with Appl. 45 (2003) 547.

[13] O. O. Olanegan, D. O. Awoyemi, B. G. Ogunware \& F. O. Obarhua, "Continuous double-hybrid point method for the solution of second order ordinary differential equations", Int. J. of Adv. Sci. \& Tech. Res. 5 (2015) 549.

[14] A. O. Adesanya, M. R. Odekunle \& A. A. James, "Order Seven Hybrid Methods for the Solution of First Order Ordinary Differential Equations", Canada Journal on Science and Engineering Mathematics 3 (2012) 154.

[15] T. A. Anake, Continuous Implicit Hybrid One-Step Methods for Solutions of Initial Value Problems of General Second Order Ordinary Differential Equations, Ph.D Thesis, Covenant University, Ota, Nigeria, 2011.

[16] Y. S. Awari, "Derivation and Application of Six-Point Linear Multistep Numerical Method for Solution of Second Order Initial Value Problems", IOSR Journal of Mathematics 7 (2013) 23. 